\author{
REVISTA SABERES EDUCATIVOS \\ DICIEMBRE 2018, $\mathrm{N}^{\circ}$ 2, 02-12
}

\title{
EL LUGAR DE LA CREATIVIDAD EN UNA DIDÁCTICA DE LA ASIGNATURA DE ARTES VISUALES EN EDUCACIÓN MEDIA
}

\author{
María Belén Carrasco Garlick ${ }^{1}$
}

\section{RESUMEN/ABSTRACT}

El siguiente artículo tiene por objetivo analizar el papel del/la docente como intermediario/a del currículo, el diseño pedagógico y los/as estudiantes en la asignatura de Artes Visuales en Educación Media. A partir de algunas observaciones de clases y a través de notas de campo, se levanta la siguiente pregunta: ¿qué debería ocurrir en la clase de Artes Visuales? Posteriormente, se realiza una revisión crítica del currículo propuesto por el Ministerio de Educación (Mineduc) para esta asignatura. Finalmente, se sugieren algunas nociones fundamentales para orientar una didáctica en este ámbito de aprendizaje, considerando una perspectiva crítica que entiende la pedagogía como un proceso creativo y al docente como creador y facilitador de atmósferas creativas.

Palabras claves: currículo; artes visuales; didáctica; proceso creativo; atmósferas creativas.

The following article aims to analyze the role of the teacher as intermediary of curriculum, pedagogical design and students in the subject of Visual Arts in high school. From some inclass observations and taking in account field notes a question is raised: What should happen on Visual Arts classes? Subsequently, a critical review of the curriculum of the subject of Visual Arts proposed by the Ministry of Education of Chile is made. Finally, some fundamental concepts in order to guide didactics for the subject of Visual Arts in high school are suggested, considering a critical perspective that understand pedagogy as a creative process and the teacher as a creator and facilitator of creative atmospheres.

Keywords: curriculum; visual arts; didactics; creative process; creative atmospheres.

\footnotetext{
${ }^{1}$ Docente de Artes Visuales, Colegio Lorenzo Sazié, Chile; Docente del Seminario de Vinculación con el medio y Habilidades Docentes, Instituto Profesional Arcos, Chile; elen.carrascoga@gmail.com.
} 


\section{Algunas VIVEnCIAS EN EL AULA QUE INVITAN A LA REFLEXIÓN}

La reflexión propuesta en este artículo tiene su origen en las primeras experiencias pedagógicas formativas promovidas desde el programa de pedagogía impartido por el Departamento de Estudios Pedagógicos (DEP) de la Universidad de Chile. Este programa incentiva a los profesores en formación a investigar con una mirada crítica las prácticas de otros/as docentes de la misma disciplina y su contexto.

En vistas de articular una reflexión sobre las prácticas pedagógicas de aula, se ha seleccionado el registro de algunas notas de campo levantadas por estudiantes participantes de este programa. Manteniendo la singularidad de los acontecimientos relatados, estas notas funcionan como dispositivos reflexivos que permiten abrir interrogantes, discusiones y posibles lineamientos para una didáctica de las artes visuales.

Alrededor de las 8:25 am la profesora comenta contenidos abordados en la sesión anterior, como el concepto de "achurado". Un estudiante dice "¿cuándo habló de eso?", mientras otro estudiante comenta "quiero que sean la una para irme a la casa".

Alrededor de las 8:40 am la profesora da las primeras indicaciones del trabajo: "Pueden hacer un dibujo libre, figurativo o abstracto, pero deben achurar. Este trabajo lo deben terminar en la primera parte de la clase, ya que es el trabajo que no terminaron o no hicieron la clase pasada".

La profesora le pregunta a un estudiante por su hoja de block, él le responde que no tiene, entonces, ella se acerca y le entrega una hoja explicando al resto de los estudiantes que tienen que traer sus materiales, ya que ella no puede estar entregándoles hojas siempre. Mientras uno de ellos le dice "pero tía es que ya estoy chato de dibujar, es más aburrido".2

\footnotetext{
${ }^{2}$ Nota de campo no 1 (2015), R. Palma, Recopilador. Santiago. Relato recuperado de la nota de campo de estudiante en práctica de la asignatura de Artes Visuales del Programa de Poslicenciatura en Educación Media
} 
La profesora explica el trabajo, dice que deben hacer un margen de dos centímetros en una hoja de block con un lápiz mina, y dentro de este margen dividir la hoja en cuatro cuadrados y un rectángulo. En cada cuadrado deben hacer "achurados" con diferentes líneas y en el rectángulo dibujar una botella achurada. La docente pregunta a los estudiantes: “¿quiénes trajeron materiales?”. Los alumnos no responden. "Ya llevan dos clases con esta actividad. No pueden comenzar cada clase desde cero. Deben traer sus materiales", agrega la profesora. Además, anuncia que va "anotar" en el libro de clases a quienes no hayan cumplido con este requerimiento. Varios alumnos se acercan pidiéndole permiso para ir a comprar una hoja de block y otros le preguntan a la profesora si ella les puede prestar un lápiz mina. La profesora me señala que "hay que venir siempre preparado, porque estos alumnos nunca traen nada". En el transcurso de la clase, un grupo de alumnas se peinan, otros escuchan música con audífonos, maniobran sus celulares y conversan entre ellos. ${ }^{3}$

A simple vista estas dos situaciones dan cuenta de ciertas convergencias en las prácticas docentes y en las conductas de los/as estudiantes. Sin ánimo de hacer extensivas estas prácticas a todos los contextos de aula, la invitación es a considerar las cualidades de los acontecimientos que posibilitan el análisis hermenéutico, y plantear la pregunta por el sentido y propósito de la enseñanza y el aprendizaje en Artes Visuales. Interrogarse por lo que sucede o debiese suceder es un punto de partida que implica observar algunas aristas asociadas al desarrollo y ejecución de los diseños pedagógicos: ¿cómo se apropia la práctica pedagógica de los propósitos curriculares impulsados por el Ministerio de Educación?; ¿cómo se genera el diseño de la experiencia en el aula?; ¿qué y quiénes están considerados/as como parte de este diseño?; ¿qué factores contribuyen al desarrollo del

en Artes Visuales de la Universidad de Chile, egreso 2016. La observación de clases se realizó en un establecimiento educacional de la comuna de Cerrillos de la Región Metropolitana, el día viernes 10 de abril del año 2015. El curso observado corresponde a un primer año de Educación Media.

${ }^{3}$ Nota de campo $\mathrm{n}^{\circ} 2$ (2015), M. Carrasco, Recopilador, Santiago. Relato recuperado de nota de campo de estudiante en práctica de la asignatura de Artes Visuales, del Programa de Poslicenciatura en Educación Media en Artes Visuales de la Universidad de Chile, egreso 2016. La observación de clases se realizó en un liceo municipal de Santiago, el día viernes 4 de mayo del año 2015. El curso observado corresponde a un primer año de Educación Media. 
diseño de esta experiencia?; y finalmente, ¿cómo realizar prácticas pedagógicas más auténticas en las cuales el proceso de enseñanza-aprendizaje tenga un sentido y propósito para todas las partes involucradas?

\section{EL CURRÍCULO DE ARTES VISUALES EN EDUCACIÓN MEDIA}

Los modelos educativos, de origen europeo y norteamericano, según los cuales se ha desarrollado la educación en Chile, han dado lugar a un proyecto educativo incompleto y sin una intención política clara de nación, carente de sentido y subordinado a un fin utilitario, declaran Gaete, Miranda y Ramírez (2007:105-106). Son estos los modelos que permearon la asignatura de Artes Visuales, la cual durante el siglo XIX tuvo una tendencia a incorporar la apreciación estética como perspectiva fundamental. Más adelante, desde inicios del siglo XX, se incentivó un fuerte predominio de la enseñanza de las Artes desde una perspectiva funcional, lo que provocó que la asignatura tuviera una condición secundaria, subordinada, menoscabada en el sistema escolar.

Con la reforma educacional del año 1990, se remplaza el nombre de la asignatura de Artes Plásticas por el de Artes Visuales y se da énfasis a la educación estética, a la sensibilidad, a la creación, a la percepción y a la reflexión crítica sobre el arte. Este impulso ha permitido que en el currículo de Artes Visuales exista una gran diversidad de estrategias y procedimientos para explorar los contenidos. En él, a partir de ejemplos de actividades de carácter optativo, se ofrecen alternativas para cumplir los objetivos fundamentales y contenidos mínimos, posibilitando, de esta manera, que el/la docente explore diferentes caminos, materiales y técnicas. El programa promueve, en este sentido, la modalidad de construcción de proyectos que permitirían integrar los intereses y habilidades de los/as estudiantes y las posibilidades que da su realidad escolar, incentivando de esta forma la motivación, la difusión de experiencias y el interaprendizaje. Asimismo, el currículo pide desarrollar en los estudiantes la expresión, la investigación teórico/práctica de la historia del arte, el diseño y la artesanía, relevando las diversas formas en que ocurre la creación artística (Errázuriz, 2001). 
A pesar de lo positiva que podría resultar esta situación, Luisa Miranda y Marcelo Espinoza (2015) entregan otra perspectiva de la situación y recalcan que en

(...) el sector de educación artística, la reforma mantuvo optativa la asignatura en la enseñanza media, y liberalizó los contenidos a tal punto que, en la actualidad, resulta difícil entender cuál es el enfoque disciplinar y didáctico que el nuevo currículo ofrece para la enseñanza de la disciplina (...). [La] fuerte tendencia al individualismo decantó en la desvalorización de la asignatura, puesto que esta debía atender a la libre expresión de los y las estudiantes, afectando las estrategias de evaluación y agencia didáctica de los docentes, y posicionándola dentro de una función recreativa más que formativa. Con ello, su valorización en los ambientes escolares industrializados decayó, ya que en estos entornos se le da valor a lo útil, evaluable y rentable.

En atención a esta misma situación, Daniela Cobos (2010), sobre la base de una encuesta realizada a profesores de Artes Visuales de colegios particulares y particulares subvencionados, aporta otros antecedentes y refuerza lo señalado por Miranda y Espinoza (2015). Cobos observa que los docentes encuestados reconocen que, a pesar de que los planes de estudios son anacrónicos, los dejan actuar con libertad, pues consideran que el currículo chileno es flexible y da la posibilidad a los establecimientos educacionales de elaborar sus propios programas de estudio. Los profesores señalan que el currículo "aunque permite que los profesores actúen con libertad, muchos no comprenden esta libertad y siguen al pie de la letra lo que dicen los planes y programas, perdiendo la capacidad creativa del profesor, desmotivando a los estudiantes". También afirman que los planes y programas "son muy idealistas, en la práctica no se pueden ejecutar tal cual proponen las actividades" y, además, “están descontextualizados y no corresponden a la realidad del país, sobre todo en los contenidos y habilidades que se espera desarrollar y los recursos de los que se supone disponen los docentes".

La autora concluye que los/as docentes encuestados/as, en general, se sienten responsables del éxito o fracaso de la asignatura, pero consideran que el Estado es "responsable político del abandono de la asignatura en el currículo escolar". 


\section{NOCIONES FUNDAMENTALES PARA UNA DIDÁCTICA DE LAS ARTES VISUALES}

En este escenario de anacronismo y paradojas del currículo, la flexibilidad de los planes y programas puede ser una oportunidad que el contexto ofrece para la implementación de una didáctica que posibilite prácticas y aprendizajes auténticos y transformadores.

A continuación se presentarán diversas propuestas que contienen nociones fundamentales para una didáctica de las Artes Visuales que oriente las prácticas pedagógicas concretas.

\section{LA PEDAGOGÍA COMO PROCESO CREATIVO}

Díaz y McKenna (2011:14) proponen que la pedagogía como proceso creativo se fundamenta en "entender a la enseñanza y el aprendizaje de nuevas maneras y formas, de formas vivas y dinámicas que siguen evolucionando tal como la sociedad y la cultura sigue evolucionando". Para reforzar esta idea, Díaz y McKenna (2011:14) citan a Maclntryre Latta (2014), quien plantea que finalmente "el currículo es un medio artístico para la construcción de sentido, una idea que añade más a esa imagen del profesor como artista. La creatividad, entonces, se convierte en uno de los resultados del aprendizaje de los estudiantes".

Díaz y McKenna, a través del relato de las experiencias del Programa de Formación Continua de Artes Integradas para Docentes realizado en la Universidad de Lesley en Estados Unidos, sostienen que la experimentación vivencial de diferentes disciplinas artísticas más la promoción de la pedagogía como proceso creativo, permiten dar cabida a la creación de lecciones innovadoras. Las autoras rescatan cuatro componentes que participan activamente en la producción de este proceso creativo: "la creación de un espacio seguro para asumir riesgos, la exploración de posibilidades, el diseño y construcción del conocimiento y la participación crítica”. (2011:1) 


\section{EL/LA DOCENTE COMO CREADOR/A}

En el programa mencionado los/as docentes son capacitado/as a través de la exploración de diferentes disciplinas artísticas, para hacer conexiones con su propia práctica profesional, incentivándolos/as a generar procesos creativos dentro de un ambiente seguro, a modo de laboratorio, donde puedan imaginar, innovar y expresar sus aprendizajes en formas artísticas. Bajo esta mirada, son considerados/as agentes creativos: se "promueve el sentido de posibilidad; el aprendizaje de nuevas capacidades dentro de sí mismo les ayuda a encontrar maneras de fomentar lo mismo en los estudiantes” (Díaz y McKenna, 2011:3). Además, dentro de este proceso los/as docentes reconocen los espacios artísticos cotidianos de la comunidad en la cual trabajan (arquitectura, teatro, murales, etc.) como potenciales lugares educativos.

El programa promueve el diseño y construcción de obras de arte o una investigación sobre temas relevantes de la sociedad contemporánea por parte de el/la docente, incluyendo diversos referentes y modalidades artísticas, a partir de los cuales se puede vivenciar en carne propia el proceso creativo. Finalmente, los/as docentes participan de un proceso evaluativo que les permite aprender a autoevaluarse y a evaluar a las demás personas, incentivando una capacidad crucial en el proceso creativo: la metacognición para crear y construir, a partir del diálogo, su propio aprendizaje.

En la misma línea, María Acaso y otros autores (2011:9), plantean que "diseñar y desarrollar proyectos educativos tiene la misma complejidad y se puede alcanzar el mismo grado de placer que cuando diseñamos un proyecto artístico”, pues ambos son generadores de conocimiento y procesos intelectuales. "Un profesor es a la vez un artista, un artista es a la vez un profesor, un estudiante es a la vez un espectador, un espectador es la vez una estudiante, un proyecto educativo es a la vez una obra de arte y una obra de arte es a la vez un proyecto educativo" (Acaso, Belver, Nuere, Moreno, Antúnez \& Ávila 2011:9). Asimismo el docente sería un proyectista, un diseñador de proyectos y un facilitador de causalidades completadas a través de las conexiones que realizan sus estudiantes. 


\section{EL/LA DOCENTE COMO FACILITADOR/A - MEDIADOR/A DE ATMÓSFERAS CREATIVAS}

En el marco de esta propuesta, el/la docente no enseñaría la creatividad, sino que la propiciaría a partir de una atmósfera creativa.

Este clima creativo debe trascender el aula y ser parte de una atmósfera de la institución escolar en su conjunto, para favorecer una actitud reflexiva y creativa ante el conocimiento, a partir de la idea de que el alumno que está aprendiendo no es el que está bien informado sino quien constantemente problematiza el conocimiento que está construyendo y creando, o sea, como un actor del conocimiento y no como un mero espectador de este. (Betancourt y Valadés, 2009:3)

Según Betancourt y Valadés (2009:3), las atmósferas creativas se nutren de tres elementos:

- El ámbito psicosocial, que hace referencia al ambiente donde las relaciones e interacciones generan "seguridad, confianza, autonomía y libertad" promoviendo una vivencia reflexiva e incitando la capacidad de asombro, el interés y la atención de los alumnos, en un espacio donde pueden compartir sus experiencias e integrarlas con los conocimientos propuestos por el/la docente. (Betancourt y Valadés, 2009:3)

- El ámbito didáctico, que releva “el cuestionamiento, el planteamiento y reformulación de problema, la inteligencia y la creatividad compartida". Se propone desafiar a la/el estudiante para que "aprenda a aprender a la vez que comprende sus procesos cognoscitivos y metacognitivos (estrategias de aprendizaje), y lo pueda unir a su vida cotidiana”. (Betancourt y Valadés, 2009:3)

- La existencia de instalaciones físicas que incentivan el enseñar y aprender.

Una atmósfera creativa desafía el desarrollo de las capacidades de los sujetos, situando el aprendizaje en diálogo permanente con su contexto histórico y cultural. Se abre a preguntas más que a respuestas y hace del error parte del proceso. Además, integra el 
afecto y el intelecto y promueve la motivación intrínseca, al reconocer y estimular los intereses de los/as estudiantes, rescatar lo lúdico y fomentar el trabajo grupal, cooperativo y colaborativo (Betancourt y Valadés, 2009:5).

\section{REFLEXIONES FINALES}

A lo largo del presente artículo se han analizado brevemente los lineamientos del currículo de Artes Visuales, diagnosticando algunas de sus posibilidades y complejidades, a partir de diferentes perspectivas. Entre ellas se destacó, en primer lugar, la diversidad de enfoques y objetivos, muchos de estos anacrónicos, que conviven en la asignatura; en segundo lugar, su carácter temático, que permitiría mayor flexibilidad para la creación pedagógica de el/la docente; y, finalmente, su segregación al poseer menos horas lectivas y tener un enfoque funcionalista.

En una segunda instancia, tras este análisis, se propusieron algunas nociones fundamentales para una didáctica de las Artes Visuales que pudiera promover prácticas concretas basadas principalmente en tres puntos: primero, considerar la pedagogía como un proceso creativo en el cual el/la docente incentiva un currículo vivo, diseñando experiencias pedagógicas que tienen en cuenta el currículo, los/as estudiantes y su contexto; segundo, considerar a el/la docente como un/a creador/a, a partir de la idea de que cuando el/la docente aprende nuevas capacidades y vivencia sus procesos creativos conscientemente, puede fomentar lo mismo en sus estudiantes; tercero, considerar a el/la docente como un/a facilitador/a - mediador/a de atmósferas creativas, enfatizando que no se enseñar la creatividad, sino que se la propicia incentivando diversas actitudes y características en el aula.

En síntesis, respecto a la preocupación por el sentido del proceso educativo situado en las Artes Visuales, es necesario considerar la formación de los/as docentes en habilidades creativas para el desarrollo de didácticas innovadoras que potencien estas cualidades en sus estudiantes, lo que permitiría reivindicar las artes en sus contextos. 
También es necesario considerar la formación de comunidades de aprendizajes o dispositivos colaborativos críticos de las prácticas pedagógicas, de manera de relevar el saber docente, para dar paso a una didáctica de las Artes Visuales que permita realizar diseños pedagógicos con sentido para todos/as los/as participantes del proceso de enseñanza-aprendizaje.

\section{REFERENCIAS}

Acaso, M., Belver, M. H., Nuere, S., Moreno, M. C., Antúnez, N., \& Ávila, N. (2011). Didáctica de las artes y la cultura visual. Madrid: Akal.

Betancourt Morejón, J., \& Valadés Sierra, M. (2009). ¿Cómo propiciar atmósferas creativas en el salón de clase? Revista Digital Universitaria, 10(2): s/p. Recuperado de $<$ http://www.revista.unam.mx/vol.10/num12/art85/int85.htm>

Cobos Bustamante, D. (2010). Innovación y creatividad en las artes visuales: las claves de la reivindicación. CIDD II Congrés Internacional de Didàctiques. Recuperado de http://www2.udg.edu/portals/3/didactiques2010/guiacdii/ACABADES\%20FINALS /284.pdf

Díaz, G., \& McKenna, M. B. (2011). Creative Process as Pedagogy. Budapest, HG: International Society for Education in the Arts (InSEA). Recuperado de http://ieie.udistrital.edu.co/pdf/El\%20proceso\%20creativo\%20como\%20pedagogia \%202012.pdf

Errázuriz, L. H. (2001). La Educación Artística en el sistema escolar chileno. Paper presentado en la Reunión Regional de la UNESCO de Expertos en Educación Artística escolar en Latinoamérica y El Caribe, Brasil. Recuperado de http://www.unesco.org/new/fileadmin/MULTIMEDIA/HQ/CLT/CLT/pdf/Arts_Edu _CaseStudies_LatinCarib_Hernan.pdf

Gaete, M., Miranda, L., Ramírez, M. (2007). Arte y Filosofía en el Currículo escolar: entre el desarraigo y el olvido. Santiago: Facultad de Filosofía y Humanidades, Universidad de Chile. 
Miranda, L. y Espinoza, M. (2015). El currículo de artes visuales en la educación chilena. Docencia (57), $17-27$. Recuperado de http://revistadocencia.cl/ revist37/web/images/ediciones/Docencia_57.pdf 